\title{
TOPIC: ABDOMINAL WALL HERNIA - Spigelian hernia, anatomy, incidence, repair
}

\author{
VS:06 \\ LAPAROSCOPIC APPROACH \\ TO THE MANAGEMENT OF SPIGELIAN HERNIA: \\ OUR EXPERIENCE \\ A Bhardwaj', S Kalhan, P Bhatia, M Khetan, S John, \\ V Bindal, J Bhat, S Wadhera, P Arora \\ 'Institute of Minimal Access Surgery-Sir Gangaram Hospital, \\ New Delhi, India
}

Introduction: Spigelian hernias, also known as 'hernia of the semilunar line', are rare primary ventral hernias arising in the Spigelian, or semilunar line located at the lateral border of rectus. As it is difficult to recognize clinically, its true incidence is probably greater than the relatively small number of patients reported in the literature. Traditional repair of such hernias use the open approach. Herein we report laparoscopic management of 7 cases of Spigelian hernias.

Methods: From March 2011 and August 2013, 7 patients underwent repair of spigelian hernia using the laparoscopic transabdominal preperitoneal technique of mesh reinforcement and reperitonization, in a single surgical unit by multiple surgeons. Diagnosis was made on the basis of clinical symptoms and radiological imaging. Our standard technique includes a three-port approach with creation of a pre-peritoneal flap $2.5 \mathrm{~cm}$ above the defect to the medial umbilical ligament, reduction of sac, extending flap till belly of psoas muscle, placement of $15 \times 15 \mathrm{cms}$ heavyweight polypropylene mesh in the space and fixation to the Cooper's ligament with tacks.

Results: Seven patients, 4 female and 3 male, underwent laparoscopic repair of Spigelian hernias. Patients' mean age was 42 years (range 33 to 65). All patients presented with symptoms of abdominal pain and palpable swelling was noted in 4 patients. Three patients had history of prior surgery but none had incisional hernia. USG/CT scan aided the diagnosis of the condition. All the patients had an uneventful post-operative recovery and were discharged on first or second post-operative day depending on comfort level. Patients were followed up at 1 week, 1 month, 6 months and then annually till date with satisfactory resolution of preoperative symptoms and no reported recurrence of hernia.

Conclusion: Spigelian hernia requires a high index of suspicion, given the lack of consistent symptoms and signs, thus needing a combination of history, physical examination, and preoperative imaging to secure the diagnosis. Laparoscopic transabdominal preperitoneal repair is safe, feasible and the modality of choice for spigelian hernia by experienced laparoscopic surgeons. We present a short video of our technique.

\section{VS:07}

\section{AN UNUSUAL PRESENTATION OF A SPIGELIAN HERNIA AND EMERGENCY LAPAROSCOPIC REPAIR WITH GOOD OUTCOME \\ M Saeed $^{1}$, S Anwar $^{1}$ \\ ${ }^{I}$ Calderdale and Huddersfield NHS Trust, Huddersfield, UNITED KINGDOM}

This is a video demonstration of a patient who presented with an incarcerated lateral abdominal wall hernia. CT scan showed this to be a spigelian hernia. This was unusually wide mouthed and caused clinical confusion. Laparoscopy showed this to be a spigelian hernia. The hernia was repaired preperitoneally with a composite mesh. The patient was discharged within 48 hours.

There are hardly any reports of obstructed spigelian hernia's in the literature. This was an unusual case treated laparoscopically. 